\title{
Synergistic effect between polyhedral oligomeric silsesquioxane and flame retardants
}

\author{
B. Safarikova ${ }^{\mathrm{a}}$, A. Kalendova, ${ }^{\mathrm{a}, \mathrm{b}}$, V. Habrova ${ }^{\mathrm{c}}$, S.Zatloukalova $^{\mathrm{a}}$ and M. \\ Machovsky $^{\mathrm{b}}$ \\ ${ }^{a}$ Department of Polymer Enginering, Faculty of Technology, Tomas Bata University in Zlin, Nam. T. G. Masaryka \\ 275, 76272 Zlin, Czech Republic \\ ${ }^{b}$ Centre of Polymer Systems, nam. T.G.Masaryka 5555, 76001 Zlin, Czech Republic, \\ ${ }^{c}$ Polymer Institut Brno, Tkalcovska 36/2, 65649 Brno, Czech Republic
}

\begin{abstract}
Synergistic effect was observed between two types of flame retardant and two types of polyhedral oligomeric silsesquioxanes (POSS). The polymer matrix was represented by low density polyethylene Bralen RB 2-62 (Slovnaft). The polyethylene was mixed with nanofillers OctaIsobutyl POSS and TriSilanolIsobutyl POSS. The flame retardants was represented by ADK STAB ${ }^{\circledR}$ FP 2200 and Exolite ${ }^{\circledR}$ APP 722 . The flammability was investigated by UL-94 test. The results showed that the APP/POSS system had important flame retardant and anti-dripping abilities for LDPE.
\end{abstract}

Keywords: LDPE, synergistic effect, flame retardant, flammability, composite PACS: 81

\section{INTRODUCTION}

Polyethylene is one of the most widespread polymers. Typically, low density polyethylene (LDPE) is used to manufacture flexible films such as those used for plastic retail bags and garment dry cleaning and grocery bags. LDPE has good isolative electrical properties and it is widely used in wire and cable applications. ${ }^{[1]}$ Further LDPE foils are used in the construction industry, where the need for increased resistance to fire. For example, higher flame resistance is necessary on the insulating films, which are used in tunnels. Therefore, it is important the development of materials with improved flame resistance, with high thermal stability.

Generally, different additives are used to modify the properties of polymers. One of the additive representatives is fillers. Fillers can be divided into natural and synthetic, by origin. Next, they can be divided into organic and inorganic, by the chemical structure. Properties of the polymers can also be influenced by the shape of the particles like spherical, cubical, irregular, block, plate, flake, fiber or mixtures of different shapes. ${ }^{[2]}$ The special type of fillers are polyhedral oligomeric silsesquioxanes (POSS). The POSS belong to organo-silicat fillers and containing silicon atoms. The incorporation of POSS molecules as nanoparticles into polymers such as polyolefins, polyesters, and polyamides are seen to bring about advantageous changes in physical and chemical properties of the polymer itself, e.g. modulus, strength, glass transition temperature, mechanical performance and thermal stability. ${ }^{[3,4,5,6]}$ Next important additive representatives is flame retardants. These additives can be divided into 4 types: halogen flame retardants, flame retardants based on phosphorus, inorganic oxides and hydroxides and retardants containing silicon. The flammability can be reduced with two mechanisms. The first mechanism is physical and the second mechanism is chemical. For example, physical mechanism is endothermic reaction, which is consuming heat and the material is so cooling.

Combustion is a thermal-oxidative reaction. Flammability is defined by a series of conditions and the combustion is undesirable reaction. Natural and synthetic polymers, when exposed to a source of sufficient heat, will decompose or "pyrolyse" evolving flammable volatiles. These mix with the air, if the temperature is high enough, ignite. ${ }^{[7]}$ Conversely, inorganic materials (f.e. glass and metal) are incombustible. Some polymers have better flame resistance than others. Some types of polymers during flammability test after the first ignition extinguishing themselves. Flammability is therefore different for different polymers depending on the structural unit constituting

Times of Polymers (TOP) and Composites 2014

AIP Conf. Proc. 1599, 106-109 (2014); doi: 10.1063/1.4876789

(C) 2014 AIP Publishing LLC 978-0-7354-1233-0/\$30.00 
the polymer. There are many tests to determine the flammability of material. One of the most famous is the test in the flame - UL 94. The test can be performed in two positions: horizontal or vertical. More frequent is using of vertical position, which is presented in this article.

This study research the synergistic effect between nanofillers and flame retardants. Rather it has been interested in the flame resistant properties of nanocomposites with polyhedral oligomeric silsesquioxanes (POSS).

\section{EXPERIMENTAL}

Low Density Polyethylene Bralen RB 2-62 from Slovnaft was used as the polymer matrix for testing. From flame retardants were used ammonium polyphosphate - Exolite ${ }^{\circledR}$ APP 722 (APP), by Clariant, and retarder based on phosphorus and nitrogen - ADK STAB ${ }^{\circledR}$ FP 2200 (ADK), by Adeka Palmalore. From silsesquioxanes were used Trisilanolisobutyl POSS (POSS 1) and Octaisobutyl POSS (POSS 2), both by Hybrid Plastics.

Polymer with the fillers and flame retardants were compounded in Brabender KO-kneader. The composition of the nanocomposites is shown in table 1 . Compounding of all samples was carried out at $130{ }^{\circ} \mathrm{C}, 110 \mathrm{rpm}$ for 20 minutes. Next prepared materials were pressed by hand press, where dimensions of mold were $125 \times 125 \times 2 \mathrm{~mm}$. The press conditions: $\mathrm{T}=150^{\circ} \mathrm{C}$, preheat 5 minutes, press 4 minutes.

The flammability test was carried out in accordance with UL 94 test. Testing was conducted in a vertical position. UL 94 is now harmonized with IEC 60707, 60695-11-10 and 60695-11-20 and ISO 9772 and $9773 .{ }^{[8]}$ Samples for measurement were cut from the plates.

The dispersion of the fillers in LDPE matrix was observed through microscopic investigations (SEM). Measurements were carried out on the unit TESCAN VEGA II. The accelerating voltage was preset to $10 \mathrm{kV}$. The pictures were taken at a magnification of $1000 \times, 5000 \times$ and $10000 \times$. Samples were prepared by breaking in liquid nitrogen. Finally the samples have been vapor-deposited conductive metal layer.

TABLE 1. Composition of the nanocomposites

\begin{tabular}{ccccc}
\hline Sample & $\begin{array}{c}\text { APP } \\
(\%)\end{array}$ & $\begin{array}{c}\text { ADK } \\
\mathbf{( \% )}\end{array}$ & $\begin{array}{c}\text { POSS 1 } \\
(\%)\end{array}$ & $\begin{array}{c}\text { POSS 2 } \\
(\%)\end{array}$ \\
\hline 1. & 26 & - & 0 & - \\
2. & 26 & - & 1 & - \\
3. & 23 & - & 3 & - \\
4. & 0 & - & 3 & - \\
5. & 26 & - & - & 0 \\
6. & 26 & - & - & 1 \\
7. & 23 & - & - & 3 \\
8. & 0 & - & - & 3 \\
$9 .$. & - & 26 & 0 & - \\
10. & - & 26 & 1 & - \\
11. & - & 23 & 3 & - \\
12. & - & 0 & 3 & - \\
13. & - & 26 & - & 0 \\
14. & - & 26 & - & 3 \\
15. & - & 23 & - & 3 \\
16. & - & 0 & - & \\
\hline
\end{tabular}

\section{RESULTS AND DISCUSSION}

Tables 2-3 are presenting the flammability by UL 94. In the table 2 are presented the UL 94 flammability for LDPE mixtures with POSS 1. 
TABLE 2. Flammability results for LDPE/APP/POSS 1 and LDPE/ADK/POSS 1

\begin{tabular}{ccc} 
Sample & Name of nanocomposite & UL 94 \\
\hline 1. & $26 \%$ APP $/ 0 \%$ POSS 1 & V-2 \\
2. & $26 \%$ APP/ $1 \%$ POSS 1 & V-0 \\
3. & $23 \%$ APP $/ 3 \%$ POSS 1 & V-1 \\
4. & $0 \%$ APP/3\% POSS 1 & Fail \\
9. & $26 \%$ ADK/0\% POSS 1 & V-0 \\
10. & $26 \%$ ADK/ $1 \%$ POSS 1 & V-0 \\
11. & $23 \%$ ADK $/ 3 \%$ POSS 1 & V-1 \\
12. & $0 \%$ ADK $/ 3 \%$ POSS 1 & Fail \\
\hline
\end{tabular}

Note: The best classification is presented by V-0.

From the table 2, it can be concluded that in sample series with POSS 1, was noted synergistic effects with flame retardant APP. The sample with pure flame retardant, $26 \%$ APP $/ 0 \% \mathrm{POSS}$, was classified as V-2. Adding $1 \%$ of the POSS 1 to the flame retardant APP was improved the classification in accordance with UL 94 from V-2 to V-0. In the mixture where the flame retardant was replaced by $3 \%$ of nanofiller, was also achieved better result. The sample was moved from class V-2 to V-1. In the samples with flame retardant ADK it has not been observed synergistic effects. Conversely, flame resistance deteriorates slightly by adding nanofiller. For sample, where the flame retardant ADK was replaced by 3\% nanofiller, was noted worsening of flame resistance in accordance with UL 94 from $\mathrm{V}-0$ to $\mathrm{V}-1$.

In the table 3 are presented the UL 94 flammability for LDPE samples with POSS 2. For samples with POSS 2, we have achieved similar results as for composition with POSS 1. Adding 1\% of the nanofiller POSS 2 to the flame retardant APP was caused improvement from V-2 to V-1. Again for the mixtures with flame retardant ADK it has not been observed improvement.

TABLE 3. Flammability results for LDPE/APP/POSS 2 and LDPE/ADK/POSS 2

\begin{tabular}{ccc}
\hline Sample & Name of anocomposite & UL 94 \\
\hline 5. & $26 \%$ APP $/ 0 \%$ POSS 2 & V-2 \\
6. & $26 \%$ APP $/ 1 \%$ POSS 2 & V-1 \\
7. & $23 \%$ APP $/ 3 \%$ POSS 2 & V-2 \\
8. & $0 \%$ APP $/ 3 \%$ POSS 2 & Fail \\
13. & $26 \%$ ADK $/ 0 \%$ POSS 2 & V-0 \\
14. & $26 \%$ ADK/ $\%$ POSS 2 & V-0 \\
15. & $23 \%$ ADK $/ 3 \%$ POSS 2 & V-1 \\
16. & $0 \%$ ADK $/ 3 \%$ POSS 2 & Fail \\
\hline
\end{tabular}

Note: The best classification is presented by $\mathrm{V}-0$. 

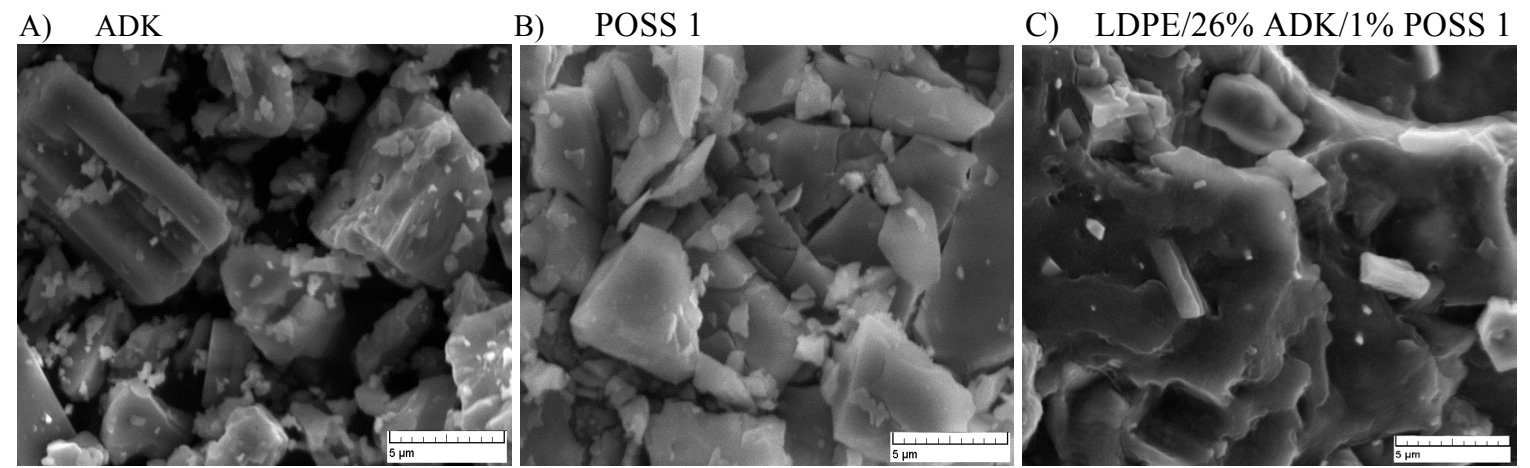

FIGURE 1. SEM Pictures of flame retardant ADK, nanofiller POSS 1 and mixture LDPE/26\%APP/1\%POSS 1

In the figure 1 are presented SEM pictures of pure flame retardant ADK (A), nanofiller POSS 1 (B) and mixture LDPE $/ 26 \%$ ADK $/ 1 \%$ POSS 1 (C). From figure $1(\mathrm{C})$ it is possible to determine, that the filler were well dispersed in the polymer matrix. Next good adhesion was detected between the filler and the LDPE. It can be also possible recognize that additives has been exhibited nanosize for some particles at least in one dimension.

\section{CONCLUSION}

For samples with flame retardant Exolite ${ }^{\circledR}$ APP 722 (APP), it was observed reductions inflammability by UL 94. Flammability reduction could be due to a synergistic effect between flame retardant APP and nanofiller POSS 1 . The samples containing only POSS failed the test UL 94, although some effect on flammability was assumed.

\section{ACKNOWLEDGMENTS}

The article was written with the support of the project FR-TI1/188 granted by Ministry of Industry and Trade of Czech Republic and the project IGA/FT/2014/014. Next this article was written with support of Operational Program Research and Development for Innovations co-funded by the European Regional Development Fund (ERDF) and national budget of Czech Republic, within the framework of project Centre of Polymer Systems (reg. number: CZ.1.05/2.1.00/03.0111).

\section{REFERENCES}

1. History of polymers \& Plastics for Teachers

Available from: http://plastics.americanchemistry.com/Education-Resources/Hands-on-Plastics/Introduction-to-Plastics-ScienceTeaching-Resources/History-of-Polymers-Plastics-for-Teachers.html

2. J. Murphy; Additives for Plastics Handbook; Elsevier Science Ltd.; Oxford 2001; ISBN 1856173704

3. E. L.Heeley; Morphology and crystalizations kinetics of polyethylene/long alkyl-chain substituted Polyhedral Oligomeric

Silsesquioxanes (POSS) nanocomposite blends: A SAXS/WAXS study; European Polymer Journal 51 (2014) 45-56

4. R. Bouza; Study of thermal and morphological properties of a hybrid system, iPP/POSS. Effect of flame retardance; Composites: Part B 58 (2014) 566-572

5. E. S. Cozza; Nanostructured nanofibers based on PBT and POSS: effect of POSS on alighnment and macromolecular orientation of the nanofibres; European Polymer Journal 49 (2013) 33-40

6. S. W. Kuo; POSS related polymer nanocomposites; Progress in Polymer Science 36 (2011) 1649-1696

7. A. R. Horrocks; Fire retardant materials; Woodhead Publishing Limited, Cambridge 2001, ISBN 1855734192

8. UL 94, The standard for safety of flammability of plastic materials for parts in devices and appliances testing

Available from: <http://www.ul.com/global/eng/pages/offerings/industries/chemicals/plastics/testing/flame/> 
AIP Conference Proceedings is copyrighted by AIP Publishing LLC (AIP). Reuse of AIP content is subject to the terms at: http://scitation.aip.org/termsconditions. For more information, see http://publishing.aip.org/authors/rights-and-permissions. 